RR $-75-16$

OPTIMAL REINSURANCE AND DIVIDEND PAYMENT STRATEGIES

Pantelis Pechlivanides

June 1975

Research Reports are publications reporting on the work of the author. Any views or conclusions are those of the author, and do not necessarily reflect those of IIASA. 


\section{Optimal Reinsurance and Dividend Payment Strategies}

Pantelis Pechlivanides ${ }^{\dagger}$

\section{Introduction}

The risk reserves* of an insurance company (I.C.) can be viewed as a random walk over time. For the example of a discrete time case, we can write

$$
R_{t+1}=R_{t}+P_{t}-\xi_{t}
$$

where

$$
\begin{aligned}
& R_{t}: \text { the risk reserves at start of period } t ; \\
& P_{t}: \text { the premiums collected during period } t ; \\
& \xi_{t}: \text { the claims during period } t \text { (a random variable). }
\end{aligned}
$$

At the end of each year, the I.C. must decide how much of the risk reserves to pay out as dividend. They must also decide how to reinsure, this being a way to transform the risk the company carries by sharing it with another company.

The problem resembles the consumption-investment problems considered in the literature connected with securities markets, and thus inspired the approach used in this study.

In the following, we will consider an I.C. with utility over time represented by the discounted sum of utilities of

†University of California, Berkeley, California, U.S.A. This paper is based upon a $\mathrm{Ph}$. D. thesis to be submitted to the University of California, Berkeley.

* The term "risk reserves" loosely refers to the reserve capital kept by a company to face claims. In other words, it is the working capital. 
each period.** The dynamic programming relation for the Nperiod problem will then be formulated. In section III, we will find a closed-form solution for a class of utility functions known as the Linear Risk Tolerance class (this will be defined later). The solutions will indicate that dividend payments should be proportional to risk reserves.

The form of the reinsurance treaty will be independent of the company's wealth. It will depend on its utility function, the price of reinsurance, and the probability density function of the claims $(\xi)$. The wealth of the company will only determine the amount of reinsurance.

These results and some generalizations will be discussed in section IV.

II. The Model

A. Description of the Insurance Company

The I.C. is faced with an N-period problem. We will count the periods backwards and call the interval $(t, t-1)$, the $t^{\text {th }}$ period. Thus, $(1,0)$ is the first period (but is in reality the last).

The relevant variables are:

$P_{t}$ : the premium collected during period $t_{i}$ (for simplicity this is assumed to be collected at the end of the period);

$\xi_{t}$ : the claims during period $t$, a positive random variable over the interval $x_{t}$, whose value will be $x_{t}$; (for simplicity this is assumed to be realized at the end of the period);

$c_{t}$ : the dividend payment at the start of period $t$ for use in period $t+1$;

* Meyer [2] has shown that other forms for evaluating consumption programs might be appropriate; we will consider such cases in Section IV. 
$R_{t}$ : the risk reserves level at the start of period $t$ before dividends are paid;

$\phi_{t}(x)$ : the probability density function of the $r \cdot v \cdot \xi_{t^{\prime}}$ assumed to exist for $\mathrm{x} \varepsilon \mathrm{x}_{t}$.

B. Utility Function of the I.C.

We assume that the I.C. can express preferences over streams of dividend payments $\underline{c} \equiv\left(c_{N}, c_{N-1}, \ldots, c_{1}, c_{O}\right)$; it does so by using a discounted form of the utilities of each period.

$$
\mathrm{U}(\underline{\mathrm{c}})=\sum_{\mathrm{k}=0}^{\mathrm{N}} \alpha^{\mathrm{k}} \mathrm{u}\left(\mathrm{c}_{\mathrm{N}-\mathrm{k}}\right), \quad 0<\alpha<1 \text {. }
$$

Thus for uncertain dividend streams, the I.C. expresses preferences by looking at the expected value of $U(\underline{c})$.

Remark: As previously stated, Meyer [2] has shown that forms other than Eq. (1) might be appropriate. Specifically, he showed that if the individual (or company) decides on future consumption streams independently of the (its) past consumption, the only utility functions possible are:

$$
\begin{aligned}
& \text { (i) } U(\underline{c})=\sum_{k=0}^{N} u_{N-k}\left(c_{N-k}\right) ; \\
& \text { (ii) } U(\underline{c})=\prod_{k=0}^{N} u_{N-k}\left(c_{N-k}\right), u_{k}(\cdot)>0, \forall_{k} \\
& \text { (iii) } U(\underline{c})=-\prod_{k=0}^{N}\left[-u_{N-k}\left(c_{N-k}\right)\right], u_{k}(\cdot)<0, \forall_{k} .
\end{aligned}
$$

Setting $\mathrm{u}_{\mathrm{N}-\mathrm{k}}(\cdot)=\alpha^{\mathrm{k}} \mathrm{u}(\cdot)$ we again get Eq. (1) as above. Cases (ii) and (iii) will be discussed in Section IV where closed-form results will be found for some one-period utility functions. 


\section{Reinsurance}

Let us assume that there is a reinsurer who is ready to accept any risk for the appropriate premium. The way in which he quotes premiums is as follows: for a r.v. $\psi$ (we denote its value as $y$ ) with range $Y$, he forms a price function $P_{\psi}(y)>0$, $\mathrm{y} \varepsilon \mathrm{Y}$, and the premium which he asks to assume $\psi$ is

$$
P[\psi] \equiv \int_{Y} Y_{\psi}(y) d y
$$

If $\mathrm{P}[\psi]$ does not exist then the reinsurer does not accept the risk $\psi$. In the following we will assume that the risks are accepted by the reinsurer, or that the above integral exists.

As a marginal case $P[1]$ is the money the reinsurer is required to pay $\$ 1$ at the end of the period of the cedent, for certain.

$$
\int_{Y} P_{\psi}(y) d y=\pi<1 \text {. }
$$

In other words $\frac{1-\pi}{\pi}$ is the interest rate, and since this must be the same for any $\psi, E q .(3)$ is a normalization condition.

Similar to expectations in probability, one can show that if $\psi=\mathrm{z}(\xi)$, then

$$
\int_{Y} \mathrm{yP}_{\psi}(\mathrm{y}) \mathrm{dy}=\int_{X} \mathrm{Z}(\mathrm{x}) \mathrm{P}_{\xi}(\mathrm{x}) \mathrm{dx}
$$

where $\mathrm{P}_{\xi}(\mathrm{x})=\mathrm{P}_{\psi}(\mathrm{z}(\mathrm{x}))\left|\mathrm{Z}^{\prime}(\mathrm{x})\right|$ by a change of variables.*

*A way to think of $\mathrm{P}_{\psi}(y)$ is as a distortion of $\phi_{\psi}(y)$, say $\mathrm{P}_{\psi}(y)=\mathrm{f}_{\psi}(y) \phi_{\psi}(\mathrm{y})$; For example, if $\mathrm{f}_{\psi}(\mathrm{y})=\mathrm{a}+$ by and $\psi=(\xi)^{2}$, then $f_{\xi}(x)=a+b x^{2}$; this means that whereas the reinsurer calculates the premium using the first moment for $\psi$, he uses the second moment for $\xi$. However, in standard insurance terminology, this implies that we have different calculation principles for different risks. But this is a matter of persuasive definition. 
Before we continue to formulate the decision problem of our initial I.C. let us note that

1) there are no transaction costs in reinsuring;

2) borrowing and lending rates are the same;

3) reinsurance contracts have a span of one period; i.e. at the end of the period the risks realize whatever payments to be made are made and the contract ceases to exist.

D. Dynamic Programming Formulation

We will suppose that the I.C. knows* (or is able to forecast) the sequences

$$
\left\{p_{t}\right\}_{t=N}^{l}, \quad\left\{\phi_{t}(x)\right\}_{t=N}^{l}, \quad\left\{p_{t}(x)\right\}_{t=N}^{l}
$$

The dynamic programming relation (D.P.) for the N-period problem of the I.C. is

$$
\begin{gathered}
f_{t}\left(R_{t}\right)=\sup _{c_{t} R_{t-1}(x)}\left\{u\left(c_{t}\right)+\alpha E f_{t-1}\left(R_{t-1}\left(\xi_{t}\right)\right)\right\} ; \\
0<\alpha<1,
\end{gathered}
$$

subject to

$$
\int_{t} R_{t-1}(x) P_{\xi_{t}}(x) d x=\int_{x_{t}}\left(\frac{R_{t}-c_{t}}{\pi_{t}}+p_{t}-x\right) P_{\xi_{t}}(x) d x
$$

where $\pi_{t} \equiv \int_{x_{t}}{ }^{P_{\xi_{t}}}(x) d x<1$. For convenience in the following,

* When we deal with closed-form solutions later in this paper, we will see that we only need to know the sequences $\left\{p_{t}\right\}_{t=N}^{1},\left\{\pi_{t}\right\}_{t=N}^{l}$, where $\pi_{t} \equiv \int_{x} P_{t}(x) d x$. In other words, we need a forecast of the interest rates $i_{t} \equiv \frac{1-\pi_{t}}{\pi_{t}}$. 
we will denote $P_{\xi_{t}}(x)$ as $P_{t}(x)$.

Condition (6) is the budget constraint. Since the reinsurer is ready to make any transaction using $P_{t}(x)$, we require that the reinsurance treaty $R_{t-1}(x)$ have the same value as the initial state of the I.C., that is

$$
\frac{R_{t}-c_{t}}{\pi_{t}}+p_{t}-x
$$

(Note that $R_{t}-c_{t}$ is the position after dividends are paid in period $t_{i}$ if the I.C. did not reinsure, $R_{t}-c_{t}$ would grow to $\frac{R_{t}-c_{t}}{\pi_{t}}$ by the end of the period when premiums are collected and claims realized.)

Looking now at the DP relation Eq. (5), we see that $f_{t}\left(R_{t}\right)$ is the maximum expected utility for a $t$-period problem starting with risk reserves level $R_{t}$. In each period $t$, the I.C. has to maximize over all possible functions $R_{t-1}(x)$ (the reinsurance treaty) and also decide on the dividend payment, $c_{t}$.

The boundary condition for the DP relation is

$$
\mathrm{f}_{\mathrm{O}}(\mathrm{R})=\mathrm{u}(\mathrm{R})
$$

The answer to the $N$-period problem is $E_{N}(R)$.

\section{Closed-Form Solutions}

The problem formulated in the last section cannot, in general, be solved analytically. In this section we will find closed-form solutions when we additionally assume that the one period utility function of the I.C. belongs to the Linear Risk Tolerance (LRT) class. 
The LRT class is defined as the solutions to the equation $\frac{\mathrm{u}^{\prime \prime}}{\mathrm{u}^{\prime}(\mathrm{x})}=\frac{\mathrm{e}}{\mathrm{ax}+\mathrm{b}}^{\dagger} \mathrm{e}, \mathrm{a}, \mathrm{b}$ reals, and not both $\mathrm{a}=0$ and $\mathrm{b}=0$ with $u^{\prime \prime}(x)<0$ and $u^{\prime}(x)>0$.

The solutions to Eq. (7) give rise to

I) $a \neq 0$, let $e=c a$

$u^{\prime}(x)=(a x+b)^{c}, a x+b>0, a c<0$

which in turn gives rise to the two subclasses

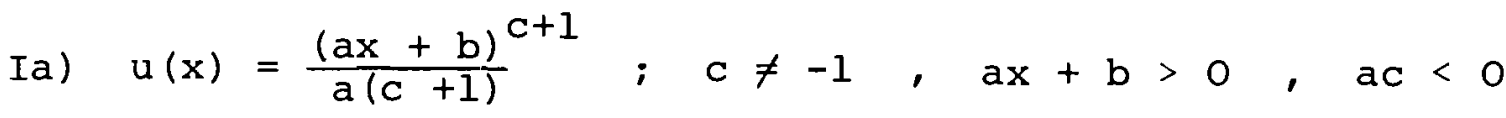

Ib) $u(x)=\frac{1}{a} \log (a x+b) ; c=-1, a>0, x>-\frac{b}{a}$

and

II) $\mathrm{a}=0$, let $\frac{\mathrm{e}}{\mathrm{b}}=-\gamma, \gamma>0$

$u^{\prime}(x)=e^{-\gamma x}$

$u(x)=\frac{1}{\gamma}\left(1-e^{-\gamma x}\right) ;-\infty<x<\infty$.

In the following we will expand on class Ia which is the richest, and will briefly mention the results for the classes Ib and II, as the arguments and derivations are analogous.

(a) Model Ia:

$$
u(x)=\frac{(a x+b)^{c+1}}{a(c+1)}, c \neq-1, a x+b>0, a c<0 \text {. }
$$

${ }^{\dagger}-\frac{u^{\prime \prime}}{u^{\prime}}$ is known as the Pratt [5] measure of risk aversion. The inverse, $-\frac{u^{\prime}}{u^{\prime \prime}}$, is called Risk Tolerance. The LRT class was investigated by Mossin [3]. 
Theorem Ia. If $u(x)$ is as above then the solution to the $t+1$ period problem described by Eqs. (5), (6) and (8) is given by:

$$
f_{t+1}\left(R_{t+1}\right)=D_{t+1} u\left(A_{t+1} R_{t+1}+B_{t+1}\right)
$$

as long as a $\left(A_{t+1} R_{t+1}+B_{t+1}\right)+b>0$, where

$$
\begin{aligned}
D_{t+1}= & 1+D_{t} \frac{m_{t+1}}{\alpha / c}, \quad D_{t+1} \geq 1 \\
A_{t+1}= & \frac{1}{D_{t+1}}, \quad 0 \leq A_{t+1} \leq 1 \\
B_{t+1}= & A_{t+1}\left[p_{t+1} \pi_{t+1}-\rho_{t+1}+\frac{b}{a A_{t}} \pi_{t+1}+\frac{B_{t}}{A_{t}} \pi_{t+1}\right. \\
& -\frac{b m_{t+1}}{\left.a A_{t}{ }^{I / c}\right]},
\end{aligned}
$$

with

$$
\mathrm{A}_{0}=1, \mathrm{D}_{0}=1, \mathrm{~B}_{\mathrm{O}}=0
$$

and $p_{t+1}, \pi_{t+1}$ defined as before

$$
\rho_{t+1} \equiv \int_{x_{t+1}} x P_{t+1}(x) d x, m_{t+1} \equiv \int_{x_{t+1}}\left(\frac{P_{t+1}(x)}{\phi_{t+1}(x)}\right)^{1 / C} P_{t+1}(x) d x
$$

The optimal dividend is

$$
c_{t+1}^{*}=A_{t+1} R_{t+1}+B_{t+1}
$$

The optimal reinsurance treaty transforms the assets of the I.C. to 


$$
R_{t}\left(\xi_{t+1}\right)=\frac{\lambda^{1 / c}}{a A_{t}}\left(\frac{P_{t+1}\left(\xi_{t+1}\right)}{\phi_{t+1}\left(\xi_{t+1}\right)}\right)^{1 / C}-\frac{b}{a A_{t}}-\frac{B_{t}}{A_{t}}, x \in X_{t+1}
$$

where

$$
\begin{aligned}
\frac{\lambda^{1 / c}}{a \bar{A}_{t}}= & \frac{1}{m_{t+1}}\left[( 1 - A _ { t + 1 } ) \left(R_{t+1}+P_{t+1} \pi_{t+1}-\rho_{t+1}+\frac{b}{a A_{t}} \pi_{t+1}\right.\right. \\
& \left.\left.+\frac{B_{t}}{A_{t}} \pi_{t+1}\right)+\frac{A_{t+1} b}{A_{t} a \alpha} m_{t+1}\right]
\end{aligned}
$$

and the solution is unique. (These results will be explained later.)

Proof. The proof first shows that the theorem holds for $t+I=1$; we then proceed by induction. Here we will expand on the proof for $t+1=1$ as the induction part is completely analogous.

Using the boundary condition (8) for the last period,

Eqs. (5) and (6) become

$$
f_{1}\left(R_{1}\right)=\max _{c_{1}, R_{0}(x)}\left[u\left(c_{1}\right)+\alpha E u\left(R_{0}(\xi)\right)\right]
$$

such that

$$
\int_{x_{1}} R_{0}(x) P_{1}(x) d x=\int_{x_{1}}\left(\frac{R_{1}-c_{1}}{\pi_{1}}+p_{1}-x\right) P_{1}(x) d x
$$

We use max instead of sup in the above since we are going to demand that the solution be interior, i.e. $a c_{1}^{*}+b>0$ and $a R_{0}(x)+b>0, x \in X$; otherwise the function $u(\cdot)$ is not well defined, at least for the case when $c<-1$.

Now we will $\mathrm{fix}_{1}$. Then the maximization of the second term in Eq. (18) subject to $\mathrm{Eq}$. (19) tells us that $\mathrm{R}_{\mathrm{O}}$ (x) must satisfy 


$$
u^{\prime}\left(R_{0}(x)\right) \phi_{1}(x)=\lambda p_{1}(x)
$$

where $\lambda$ is given by substitution in (19). This result follows from the calculus of variations. Since $u(\cdot)$ is also strictly concave Eq. (20) is necessary and sufficient and $R_{0}(x)$ is unique. Now by choice of $u(\cdot),\left(\right.$ Ia), $u^{\prime}(\cdot)$ has a range of $(0, \infty)$; thus Eq. (20) always has a solution as long as $\lambda>0$. Then

$$
R_{0}(x)=\frac{\lambda^{1 / c}}{a}\left(\frac{P_{1}(x)}{\phi_{1}(x)}\right)^{1 / c}-\frac{b}{a}
$$

while substituting Eq. (21) in (19) we have:

$$
\lambda^{1 / c}=\frac{a}{m_{1}}\left(R_{1}-c_{1}+p_{1} \pi_{1}+\frac{b}{a} \pi_{1}-\rho_{1}\right)
$$

with $\rho_{1}, m_{1}$ defined as in $(14)$. Since we require $\lambda>0$, we ask $\lambda^{1 / C}>0$ or

$$
\mathrm{a}\left(\mathrm{R}_{1}-\mathrm{c}_{1}+\mathrm{p}_{1} \pi_{1}+\frac{\mathrm{b}}{\mathrm{a}} \pi_{1}-\rho_{1}\right)+\mathrm{b} \pi_{1}>0 \text {. }
$$

Now, by substituting Eqs. (21) and (22) in (18) and manipulating, we obtain

$$
\begin{aligned}
f_{1}\left(R_{1}\right)= & \max _{c_{1}}\left\{u\left(c_{1}\right)+\frac{\alpha m_{1}}{a(c+1)}\left[\frac { a } { m _ { 1 } } \left(R_{1}-c_{1}+p_{1} \pi_{1}-\rho_{1}\right.\right.\right. \\
& \left.\left.\left.+\frac{b}{a} \pi_{1}\right)\right]^{c+1}\right\}
\end{aligned}
$$

where we have used

$$
E\left[\left(\frac{\mathrm{P}_{1}(\mathrm{x})}{\phi_{1}(\mathrm{x})}\right)^{\frac{\mathrm{C}+1}{\mathrm{C}}}\right]=\int \frac{\mathrm{P}}{\phi}\left(\frac{\mathrm{P}}{\phi}\right)^{1 / \mathrm{C}} \phi=\mathrm{m}_{1} .
$$


Now the second term in Eq. (24) is strictly concave because of Eq. (23L and the first term is strictly concave as long as $a c_{1}+b>0$. Thus, differentiating w.r.t. $c_{1}$ and equating to zero we obtain the unique solution

$$
\mathrm{C}_{1}^{*}=\mathrm{A}_{1} \mathrm{R}_{1}+\mathrm{B}_{1}
$$

with $A_{1}, B_{1}$ as defined in the statement of the theorem. Further, we find that for $\mathrm{c}_{1}^{*}$ the condition $\mathrm{ac}_{1}^{*}+\mathrm{b}>0$ is equivalent to Eq. (23). Thus the only condition needed is

$$
a\left(A_{1} R_{1}+B_{1}\right)+b>0 \text {. }
$$

Finally, by substituting $c_{1}^{*}$ in Eq. (24) we obtain

$$
f_{1}\left(R_{1}\right)=D_{1} u\left(A_{1} R_{1}+B_{1}\right)
$$

where $D_{1}$ is defined in theorem.

The induction step assumes

$$
\begin{aligned}
& E_{t}\left(R_{t}\right)=D_{t} u\left(A_{t} R_{t}+B_{t}\right), \\
& D_{t} A_{t}=1, D_{t} \geq 0, A_{t} \geq 0, a\left(A_{t} R_{t}+B_{t}\right)+b>0,
\end{aligned}
$$

and proves the result for $f_{t+1}\left(R_{t+1}\right)$. The arguments are analogous to the last-step case.

We will simply state the results for the other two models, Ib and II.

(b) Model Ib

$$
\begin{aligned}
& u(x)=\frac{1}{a} \log (a x+b) ; a>0, a x+b>0 \\
& u^{\prime}(x)=(a x+b)^{-1} .
\end{aligned}
$$


Theorem Ib. If $u(x)$ is as above then the solution to the $t+1$ period problem described by Eqs. (5), (6) and (8) is

$$
f_{t+1}\left(R_{t+1}\right)=D_{t+1} u\left(A_{t+1} R_{t+1}+B_{t+1}\right)+E_{t+1}
$$

as long as

$$
a\left(A_{t+1} R_{t+1}+B_{t+1}\right)+b>0
$$

where

$$
\begin{aligned}
& D_{t+1}=1+\alpha D_{t}, \quad D_{t+1} \geq 1 \\
& A_{t+1}=\frac{1}{D_{t+1}}, \quad 0 \leq A_{t+1} \leq 1 \\
& B_{t+1}=A_{t+1}\left[p_{t+1} \pi_{t+1}-\rho_{t+1}+\frac{B_{t}}{A_{t}} \pi_{t+1}+\frac{b}{a} \pi_{t+1}-\alpha \frac{b}{a A_{t}}\right] \\
& E_{t+1}=\frac{\alpha}{a} D_{t}\left[\log \alpha+q_{t+1}\right]+\alpha E_{t}
\end{aligned}
$$

with

$$
\begin{aligned}
& D_{0}=1, A_{0}=1, B_{0}=0, E_{0}=0 \\
& P_{t+1}, \pi_{t+1}, \rho_{t+1}
\end{aligned}
$$

defined as before, i.e.

$$
q_{t+1} \equiv E\left[\log \frac{\phi_{t+1}\left(\xi_{t+1}\right)}{P_{t+1}\left(\xi_{t+1}\right)}\right] .
$$

The optimal dividend is

$$
c_{t+1}^{*}=A_{t+1} R_{t+1}+B_{t+1} \text {. }
$$


$-13-$

The optimal reinsurance treaty transforms the assets of the I.C. to

$$
R_{t}\left(\xi_{t+1}\right)=\frac{1}{a A_{t} \lambda} \frac{\phi_{t+1}\left(\xi_{t+1}\right)}{P_{t+1}\left(\xi_{t+1}\right)}-\frac{b}{a A_{t}}-\frac{B_{t}}{A_{t}} ; \quad \xi_{t+1} \in x_{t+1}
$$

where

$$
\begin{aligned}
\frac{1}{a A_{t} \lambda}= & \left(1-A_{t+1}\right)\left(R_{t+1}+p_{t+1} \pi_{t+1}-\rho_{t+1}+\frac{b}{a A_{t}} \pi_{t+1}+\frac{B_{t}}{A_{t}} \pi_{t+1}\right) \\
& +a \frac{A_{t+1}}{A_{t}} \frac{b}{a} .
\end{aligned}
$$

Further, the solution is unique.

Proof. (omitted; similar to Theorem Ia)

(c) Model II

$$
\mathrm{u}(\mathrm{x})=\frac{1}{\gamma}\left(1-\mathrm{e}^{-\gamma \mathrm{x}}\right) ; \gamma>0,-\infty<\mathrm{x}<\infty .
$$

Theorem II. If $u(x)$ is as above then the solution to the $t+1$ period problem described by Eq. (5), (6) and (8) is

$$
f_{t+1}\left(R_{t+1}\right)=D_{t+1} u\left(A_{t+1} R_{t+1}+B_{t+1}\right)+E_{t+1}
$$

where

$$
\begin{aligned}
& \mathrm{D}_{t+1}=1+\pi_{t+1} \mathrm{D}_{t} ; \quad \mathrm{D}_{t+1} \geq 1 \\
& \mathrm{~A}_{t+1}=\frac{1}{\mathrm{D}_{t+1}} ; \quad 0 \leq \mathrm{A}_{t+1} \leq 1 \\
& \mathrm{~B}_{t+1}= \mathrm{A}_{t+1}\left[\mathrm{p}_{t+1} \pi_{t+1}-\rho_{t+1}+\frac{\mathrm{B}_{t}}{\mathrm{~A}_{t}} \pi_{t+1}+\frac{\mathrm{w}_{t+1}}{\gamma \mathrm{A}_{t}}\right. \\
&\left.-\frac{\pi t+1}{\gamma \mathrm{A}_{t}} \log \alpha\right]
\end{aligned}
$$




$$
E_{t+1}=\frac{D_{t}}{\gamma}\left[\alpha-\pi_{t+1}\right]+\alpha E_{t}
$$

with

$$
\begin{aligned}
& D_{0}=1=A_{0}, B_{0}=0, E_{0}=0 \\
& p_{t+1}, \pi_{t+1}, \rho_{t+1},
\end{aligned}
$$

as before, and

$$
w_{t+1} \equiv \int_{x_{t+1}} \log \left(\frac{P_{t+1}(x)}{\phi_{t+1}(x)}\right) P_{t+1}(x) d x .
$$

The optimal dividend is again

$$
c_{t+1}^{*}=A_{t+1} R_{t+1}+B_{t+1}
$$

The optimal reinsurance treaty transforms the assets of the I.C. to

$$
R_{t}\left(\xi_{t+1}\right)=-\frac{1}{\gamma \bar{A}_{t}} \log \left(\frac{\lambda P_{t+1}\left(\xi_{t+1}\right)}{\phi_{t+1}\left(\xi_{t+1}\right)}\right)-\frac{B_{t}}{\bar{A}_{t}} ; \xi_{t+1} \in x_{t+1}
$$

or equivalently,

$$
\begin{aligned}
R_{t}\left(\xi_{t+1}\right)= & \frac{1}{\pi t+1}\left(1-A_{t+1}\right)\left(R_{t+1}+p_{t+1} \pi t+1-\rho_{t+1}+\frac{{ }_{t+1}}{\gamma A_{t}}\right) \\
& -\frac{A_{t+1} B_{t}}{A_{t}}+\frac{A_{t+1} \log \alpha}{\gamma A_{t}}-\frac{1}{\gamma A_{t}} \log \frac{P_{t+1}\left(\xi_{t+1}\right)}{\phi_{t+1}\left(\xi_{t+1}\right)} .
\end{aligned}
$$

Again, the solution is unique. 
Proof. (omitted; similar to Ia).

IV. Remarks

1. Looking at the formulas for dividend payments Eqs. (15), (35), and (45) we see that they are linear in the risk reserves level $\mathrm{R}_{t+1}$.

However, we did not constrain the problem to $c_{t+1}^{*} \geq 0$.

Thus it is possible to have negative dividends.

Specifically, for Model Ia we have the following cases:

A. $c>0, a<0 \Rightarrow c_{t}^{*}<-\frac{b}{a}$

(a) $\quad$ b $>0 \Longrightarrow-\frac{b}{a}>0$.

Thus, $c_{t}^{*}$ can be positive or negative

(b) $b<0 \Rightarrow c_{t}^{*}$ always negative.

Case $\mathrm{Ab}$ can be viewed as a charitable organization that expects to lose all the time.

B. $\mathrm{c}<\mathrm{O}, \mathrm{a}>\mathrm{O} \Rightarrow \mathrm{c}_{\mathrm{t}}^{*}>-\frac{\mathrm{b}}{\mathrm{a}}$

(a) $\mathrm{b}>\mathrm{O} \Rightarrow \mathrm{c}_{\mathrm{t}}^{*}$ positive or negative,

(b) $b<0 \Longrightarrow c_{t}^{*}$ always positive

For the other cases, negative dividends simply mean that it is to the benefit of the stockholders to increase the capital of the I.C. in expectation of future returns, once they have decided that they will stay in business until the $\mathrm{N}$ periods have elapsed.

2. All Models can be extended to the infinite horizon stationary case simply by taking $\mathrm{N} \rightarrow \infty$.

3. The terms describing the assets of the I.C. after the reinsurance treaty can be explained for Model Ia (16), (17) as follows: 
The I.C. borrows the amount $\left(\frac{b}{a_{t}}+\frac{B_{t}}{A_{t}}\right) \pi_{t+1}$ (which grows to $\frac{b}{a A_{t}}+\frac{B_{t}}{A_{t}}$ by the end of the period) from the reinsurer. It sells its portfolio of premiums and risks and in return accepts the amount $p_{t+1}{ }_{t+1}-\rho_{t+1}$. It then forms the sum

$$
\left(R_{t+1}+p_{t+1} \pi_{t+1}-\rho_{t+1}+\frac{b}{a A_{t}} \pi_{t+1}+\frac{B_{t}}{A_{t}} \pi_{t+1}\right)
$$

from which it gives a portion $A_{t}$ (recall $0 \leq A_{t} \leq 1$ ) to the stockholders. They pay back to the I.C. the amount of

$$
\frac{A_{t+1} b m_{t+1}}{A_{t} \text { a } a^{1 / c}}
$$

(which is the cost of buying $\frac{{ }_{t+1}}{A_{t}} \frac{b}{a a^{I} / c}$ units of risky investment, $\left(\frac{P_{t+1}\left(\xi_{t+1}\right)}{\phi_{t+1}\left(\xi_{t+1}\right)}\right)^{1 / c}$.)

Then the I.C. uses what is left, i.e.

$$
\begin{gathered}
\left(1-A_{t}\right)\left(R_{t+1}+p_{t+1} \pi_{t+1}-\rho_{t+1}+\frac{b}{a A_{t}} \pi_{t+1}+\frac{B_{t}}{A_{t}} \pi_{t+1}\right) \\
+\frac{A_{t+1} b m_{t+1}}{A_{t} a \alpha^{1 / C}},
\end{gathered}
$$

to buy the risky investment at a cost of $\mathrm{m}_{t+1}$ per unit. (Similar explanations hold for Models Ib and II)

$$
\text { 4. If } \frac{P_{t+1}(x)}{\phi_{t+1}(x)} \text { increases in } x \text {, then } R_{t}(x) \text { decreases in } x
$$

for all models. This is easy to check and means that the cedent participates positively in the losses; that is, the higher the claim the lower his risk reserve level will be at the start of period $t$. 
Also, $\frac{P_{t+1}(x)}{t+1}$ increases with $x "$ indicates that the premium the reinsurer assigns to risks with high dispersion is higher than to the premium with low dispersion--this is quite reasonable.

5. A generalization of the problem can be achieved if we introduce the decision to spend money for sales promotion. It turns out that current policies do not change except for the value of $B_{t}$, the constant amount paid to the stockholders. For further details see [4].

6. Our results resemble those of Hakansson [1] for the investment consumption problem of the individual. Hakansson was able to find closed-form solutions for the one period utility functions: $u(x)=x^{\gamma}, 0<\gamma<1 ; u(x)=-x^{-\gamma}, \gamma>0$; $u(x)=\log x$ and $u(x)=-e^{-\gamma x}, \gamma>0$. Apart from deciding on his level of consumption, the individual had to decide on forming a linear combination of a fixed finite number of investment opportunities.

In our case, the company chooses the reinsurance treaty (thus its investment) for solving a calculus of variations problem, thus satisfying Eq. (20). This is exactly the reason why we were able to find closed-form solutions for a wider class (the LRT class) than that of Hakansson.

7. We will now discuss the cases when the utility of a consumption stream is not given by (i) or Eq. 1 but by cases (ii) and (iii) multiplicative forms from page 4. First we let $u_{k}(\cdot)$ $=u(\cdot), \forall k$. The D.P. relation can again be formulated as

$$
f_{t+1}\left(R_{t+1}\right)=\max _{c_{t+1}, R_{t}}\left\{u\left(c_{t+1}\right) E f_{t}\left(R_{t}\right)\right\}, u(\cdot)>0
$$

along with the budget constraint and the boundary condition

$$
f_{0}\left(R_{0}\right) \equiv u\left(R_{0}\right)
$$


where we have assumed that $u_{k}(\cdot)=u(\cdot), k=1, \ldots, N$. The formulation for $u<0$ is similar:

$$
f_{t+1}\left(R_{t+1}\right)=\max _{c_{t+1}, R_{t}}\left\{-u\left(c_{t+1}\right) E f_{t}\left(R_{t}\right)\right\}
$$

and has the same constraints and boundary condition.

We note right away that discounting has no meaning here, as it will not affect the policies. Using Eq. (48) and

$$
\begin{gathered}
u(x)={\frac{(a x+b)^{c+1}}{a(c+1)},}^{a>0,-1<c<0,} \\
a x+b>0
\end{gathered}
$$

or Eq. (49) and

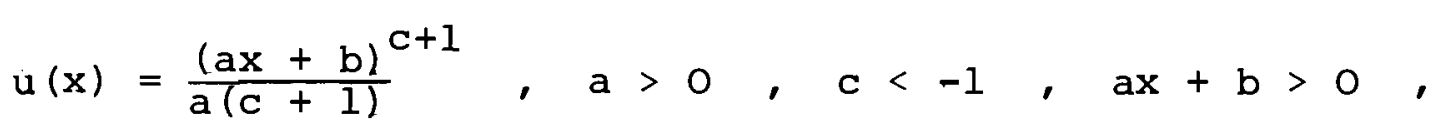

we can again find closed-form solutions to the D.P. problem of the I.C.

The solution to the $t+1$ period problem for Eqs. (48) and (5) is

$$
f_{t+1}\left(R_{t+1}\right)=D_{t+1}\left[u\left(A_{t+1} R_{t+1}+B_{t+1}\right)\right]{ }^{t+2}
$$

as long as a $\left(A_{t+1} R_{t+1}+B_{t+1}\right)+b>0$, where

$$
\begin{aligned}
& A_{t+1}=\frac{1}{t+2}, \\
& D_{t+1}=m_{t+1}{\frac{t+1}{m_{t+1}}}^{(t+1)(c+1)} D_{t}, \\
& B_{t+1}=A_{t+1}\left[p_{t+1} \pi_{t+1}-\rho_{t+1}+\frac{b}{a} \frac{\pi_{t+1}}{A_{t}}+\frac{B_{t}}{A_{t}} \pi_{t+1}-\frac{b}{a}(t+1)\right]
\end{aligned}
$$


$-19-$

where

$$
m_{t+1}=\int_{x_{t+1}}\left(\frac{P_{t+1}(x)}{\phi_{t+1}(x)}\right)^{\frac{1}{(t+1(c+1)-1}} P_{t+1}(x) d x
$$

and

$$
p_{t+1}, p_{t+1}, \pi_{t+1}
$$

are as before.

The optimal dividend payment is

$$
c_{t+1}^{*}=A_{t+1} R_{t+1}+B_{t+1}
$$

The optimal reinsurance treaty transforms the wealth of the I.C. to

$$
\begin{aligned}
R_{t}\left(\xi_{t+1}\right)= & \frac{a}{m_{t+1}}\left[\left(1-A_{t+1}\right)\left(R_{t+1}+p_{t+1} \pi_{t+1}-\rho_{t+1}+\frac{b \pi_{t+1}}{a A_{t}}+\frac{B_{t}}{A_{t}} \pi_{t+1}\right)\right. \\
& \left.+\frac{A_{t+1}}{A_{t}} \frac{b}{a}\right]\left[\frac{P_{t+1}\left(\xi_{t+1}\right)}{\Phi_{t+1}\left(\xi_{t+1}\right)}\right]^{\frac{1}{(t+1)(c+1)-1}}-\frac{b}{a A_{t}}-\frac{B_{t}}{A_{t}} .
\end{aligned}
$$

The solution is again unique.

The proof is similar to that of the discounted sum of utilities case and will not be presented here. In addition, the above solution is the same for Ers. (49) and (51), except that now

$$
\left.f_{t+1}\left(R_{t+1}\right)=(-1)^{t+1} D_{t+1}\left[u\left(A_{t+1} R_{t+1}+B_{t+1}\right)\right]\right]^{t+2} .
$$


We see that the optimal policies found for the multiplicative utility cases are essentially the same as that of the discounted sum approach. We should note, however, that $A_{t}=\frac{1}{t+1}$ (recall that $A_{t}$ is the portion of $R_{t}$ distributed as dividend). This occurs because of the form of the multiplicative utility which gives equal value to the consumption (dividend) of all periods.

We cannot argue about what happens in the limit, as $t \rightarrow \infty$ as $D_{t}$ has no limit (see Eq. (54)).

Finally, we must realize that concerning the discounted sum of utilities $\mathrm{U}=\sum_{\mathrm{k}=0}^{\mathrm{N}} \alpha^{\mathrm{k}} \mathrm{u}\left(\mathrm{x}_{\mathrm{k}}\right)$, a change of $\mathrm{u}$ to the oneperiod utility equivalent $a u+b$ produces a utility equivalent change in $U$ (to $a U+b)$; in the multiplicative utility case $\left(U=\prod_{k=0}^{N} u\left(x_{k}\right)\right.$, however, a utility equivalent change in u does not result in a utility equivalent change in $U$, i.e. $A, B$ are such that

$$
\left.\prod_{k=1}^{N}\left[a u\left(x_{k}\right)+b\right]=A \prod_{k=1}^{N} u\left(x_{k}\right)+B\right)
$$

Thus the closed-form results found for Eqs, (48) and (49) are limited to the specific utility functions (50) and (51), respectively, and do not hold for any other function that is utility equivalent to them. 


\section{$\underline{\text { References }}$}

[1] Hakansson, N. "Optimal Investment and Consumption Strategies under Risk for a Class of Utility Functions," Econometrica, Vol. 38, No. 5, pp. 587-607, (1970).

[2] Meyer, R.F. "On the Relationship Among the Utility of Assets, the Utility of Consumption, and Investment Strategy in an Uncertain, but Time-invariant, World," Proceedings of the Fifth International Conference of Operational Research, Venice, 1969.

[3] Mossin, J. Theory of Financial Markets, New York, Prentice Hall, 1973.

[4] Pechlivanides, P. (Thesis in progress) to be published with the Operations Research Center, University of California, Berkeley.

[5] Pratt, J. "Risk-Aversion in the Small and in the Large," Econometrica, January-April 1964. 
